\title{
CORRECTION
}

\section{Author Correction: Hormones induce the formation of luminal-derived basal cells in the mammary gland}

Wenqian Song ${ }^{1}$, Ran Wang ${ }^{1}$, Weimin Jiang ${ }^{1}$, Qi Yin ${ }^{1}$, Guangdun Peng $\mathbb{D}^{2,3}$, Ruikai Yang ${ }^{1}$, Qing Cissy $\mathrm{Yu}^{1}$, Jianfeng Chen ${ }^{1}$, Jingsong $\mathrm{Li}^{1}$, Tom H. Cheung ${ }^{4}$, Naihe Jing (iD) ${ }^{1}$ and Yi Arial Zeng ${ }^{1}$

Cell Research (2019) 29:338; https://doi.org/10.1038/s41422-019-0147-6

Correction to: Cell Research (2019) 0:1-15; https://doi.org/10.1038/ s41422-018-0137-0; published online 10 January 2019.
In the initial published version of this article, there was a mistake in one author name (Jingsong Li). The correct name should be "Jinsong Li". This correction does not affect the description of the results or the conclusions of this work.

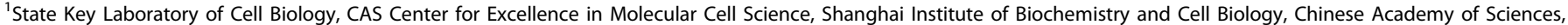

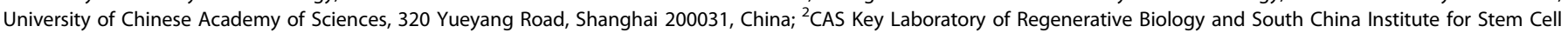

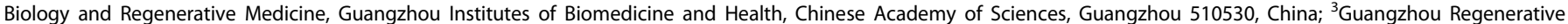

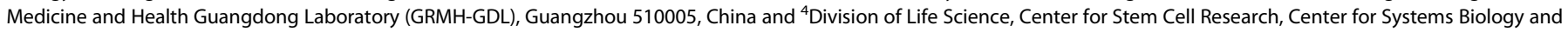
Human Health, the State Key Laboratory in Neuroscience, Hong Kong University of Science \& Technology, Kowloon, Hong Kong, China

Correspondence: Yi Arial Zeng (yzeng@sibcb.ac.cn)

These authors contributed equally: Wenqian Song, Ran Wang

Published online: 26 February 2019 